\title{
HYPERKÄHLER MANIFOLDS WITH TORSION OBTAINED FROM HYPERHOLOMORPHIC BUNDLES
}

\author{
Misha VERBITSKY
}

\begin{abstract}
We construct examples of compact hyperkähler manifolds with torsion (HKT manifolds) which are not homogeneous and not locally conformal hyperkähler. Consider a total space $T$ of a tangent bundle over a hyperkähler manifold $M$. The manifold $T$ is hypercomplex, but it is never hyperkähler, unless $M$ is flat. We show that $T$ admits an HKT-structure. We also prove that a quotient of $T$ by a $\mathbb{Z}$-action $v \longrightarrow q^{n} v$ is HKT, for any real number $q \in \mathbb{R}, q>1$. This quotient is compact, if $M$ is compact. A more general version of this construction holds for all hyperholomorphic bundles with holonomy in $S p(n)$.
\end{abstract}

\section{Introduction}

Hyperkähler manifolds with torsion (HKT-manifolds) were introduced by P.S. Howe and G. Papadopoulos ([HP]) and much discussed in physics literature since then. For an excellent survey of these works written from a mathematician's point of view, the reader is referred to the paper of G. Grantcharov and Y.S. Poon [GP]. In physics, HKT-manifolds appear as moduli of brane solitons in supergravity and M-theory ([GP2], $[\mathrm{P}])$. HKT-manifolds also arise as moduli space of some special black holes in $\mathrm{N}=2$ supergravity ([GP1], [GPS]).

The term "hyperkähler manifold with torsion" is actually quite misleading, because an HKT-manifold is not hyperkähler. This is why we prefer to use the abbreviation "HKT-manifold".

HKT-manifolds are hypercomplex manifolds equipped with a special kind of Riemannian metrics.

A hypercomplex manifold $([\mathrm{Bo}])$ is a $C^{\infty}$-manifold $M$ endowed with a triple of almost complex structures $I, J, K \in \operatorname{End}(T M)$ which are integrable and satisfy the quaternionic relations $I \circ J=-J \circ I=K$. If, in addition, $M$ is equipped with a Riemannian structure $g$ preserved by $I, J, K$, then $M$ is called hypercomplex Hermitian. If $(M, g)$ is Kähler with respect to $I, J, K$, then $(M, g, I, J, K)$ is called hyperkähler.

An HKT-manifold is a hypercomplex Hermitian manifold which satisfies a similar, but weaker condition (1.1).

Received March 16, 2003.

The author is partially supported by CRDF Grant RM1-2354-MO02 and EPSRC Grant GR/R77773/01. 
Let $(M, g, I, J, K)$ be a hypercomplex Hermitian manifold. Write the standard Hermitian forms on $M$ as follows:

$$
\omega_{I}:=g(\cdot, I \cdot), \quad \omega_{J}:=g(\cdot, J \cdot), \quad \omega_{K}:=g(\cdot, K \cdot) .
$$

By definition, $M$ is hyperkähler iff these forms are closed. The HKT condition is weaker:

$$
\partial\left(\omega_{J}+\sqrt{-1} \omega_{K}\right)=0 .
$$

Notice that $\Omega=\frac{1}{2}\left(\omega_{J}+\sqrt{-1} \omega_{K}\right)$ is a $(2,0)$-form, for any hypercomplex Hermitian manifold, as an elementary linear-algebraic calculation insures. This form is called the canonical (2,0)-form associated with the hypercomplex Hermitian structure. As we shall see (Proposition 3.2), the metric can be recovered from the hypercomplex structure and the form $\Omega$.

Originally, the HKT-manifolds were defined in terms of a quaternionic invariant connection with totally antisymmetric torsion (see [HP], [GP]).

Many homogeneous examples of compact HKT-manifolds were obtained in $[\mathrm{HP}]$ and $[\mathrm{GP}]$. In [I] it was shown that any locally conformally hyperkähler manifold also admits an HKT-structure (see [Or]). Converse result was obtained in [OPS]: it was found when an HKT-manifold is locally conformally hyperkähler manifold, in terms of symmetry conditions.

Locally, the HKT metrics can be studied using potential functions ([GP]) in the same fashion as one uses plurisubharmonic functions to study Kähler metrics. This way one obtains many examples of HKT-structures on a sufficiently small open hypercomplex manifolds.

If $\operatorname{dim}_{\mathbb{R}} M=4$, every hypercomplex Hermitian metrics is also HKT (the condition (1.1) is satisfied vacuously because the left hand side of (1.1) is a $(3,0)$-form).

If $\operatorname{dim}_{\mathbb{R}} M>4$, the HKT-condition becomes highly non-trivial. There are examples of hypercomplex manifolds not admitting an HKT-structure ([FG]). All known examples of compact HKT-manifolds are either homogeneous or locally conformally hyperkähler.

In the present paper, we construct HKT-structures on fibered spaces associated with hyperkähler manifolds. A typical example of our construction is the following

Theorem 1.1. Let $M$ be a hyperkähler manifold and

$$
T^{\circ} M=\operatorname{Tot}(T M) \backslash\{\text { zero section }\}
$$

the total space of non-zero vectors in TM. Given $q \in \mathbb{R},|q| \neq 1$, let $\sim_{q}$ be the equivalence relation generated by $x \sim_{q} q x, x \in T M$. Consider the quotient $T^{\circ} M / \sim_{q}$. Then $T^{\circ} M / \sim_{q}$ is equipped with a natural HKT-structure.

Proof. See Theorem 8.1.

Theorem 1.1 is a special case of a much more general construction performed in Section 8. 


\section{The $q$-Dolbeault bicomplex}

In this Section, we introduce some notions of quaternionic linear algebra which will be used further on. A reader well versed in quaternions can safely skip this section. We follow [V5].

Let $M$ be a hypercomplex manifold, and

$$
\Lambda^{0} M \stackrel{d}{\longrightarrow} \Lambda^{1} M \stackrel{d}{\longrightarrow} \Lambda^{2} M \stackrel{d}{\longrightarrow} \ldots
$$

its de Rham complex. Consider the natural action of $S U(2)$ on $\Lambda^{*} M$. Clearly, $S U(2)$ acts on $\Lambda^{i} M, i \leqslant \frac{1}{2} \operatorname{dim}_{\mathbb{R}} M$ with weights $i, i-2, i-4, \ldots$

We denote by $\Lambda_{+}^{i}$ the maximal $S U(2)$-subspace of $\Lambda^{i}$, on which $S U(2)$ acts with weight $i$.

The following linear-algebraic lemma allows one to compute $\Lambda_{+}^{i}$ explicitly

Lemma 2.1. In the above assumptions, let I be an induced complex structure, and $\mathbb{H}_{I}$ the quaternion space, considered as a 2-dimensional complex vector space with the complex structure induced by $I$. Denote by $\Lambda_{I}^{p, 0}(M)$ the space of $(p, 0)$ forms on $(M, I)$. The space $\mathbb{H}_{I}$ is equipped with a natural action of $S U(2)$. Consider $\Lambda_{I}^{p, 0}(M)$ as a representation of $S U(2)$, with trivial group action. Then, there is a canonical isomorphism

$$
\Lambda_{+}^{p}(M) \cong S_{\mathbb{C}}^{p} \mathbb{H}_{I} \otimes_{\mathbb{C}} \Lambda_{I}^{p, 0}(M),
$$

where $S_{\mathbb{C}}^{p} \mathbb{H}_{I}$ denotes a $p$-th symmetric power of $\mathbb{H}_{I}$. Moreover, the $S U(2)$-action on $\Lambda_{+}^{p}(M)$ is compatible with the isomorphism (2.1).

Proof. This is [V5], Lemma 8.1.

Consider an $S U(2)$-invariant decomposition

$$
\Lambda^{p}(M)=\Lambda_{+}^{p}(M) \oplus V^{p},
$$

where $V^{p}$ is the sum of all $S U(2)$-subspaces of $\Lambda^{p}(M)$ of weight less than $p$. Using the decomposition (2.2), we define the quaternionic Dolbeault differential $d_{+}: \Lambda_{+}^{*}(M) \longrightarrow \Lambda_{+}^{*}(M)$ as a composition of de Rham differential and projection of to $\Lambda_{+}^{*}(M) \subset \Lambda^{*}(M)$. Since the de Rham differential cannot increase the $S U(2)$-weight of a form more than by $1, d$ preserves the subspace $V^{*} \subset \Lambda^{*}(M)$. Therefore, $d_{+}$is a differential in $\Lambda_{+}^{*}(M)$.

Let $M$ be a hypercomplex manifold, and $I$ an induced complex structure. Consider the operator $\mathcal{I}: \Lambda^{*}(M) \longrightarrow \Lambda^{*}(M)$ mapping a $(p, q)$-form $\eta$ to $\sqrt{-1}(p-$ q) $\eta$. By definition, $\mathcal{I}$ belongs to the Lie algebra $\mathfrak{s u}(2)$ acting on $\Lambda^{*}(M)$ in the standard way. Therefore, $\mathcal{I}$ preserves the subspace $\Lambda_{+}^{*}(M) \subset \Lambda^{*}(M)$. We obtain the Hodge decomposition

$$
\Lambda_{+}^{*}(M)=\oplus_{p, q} \Lambda_{+, I}^{p, q}(M) .
$$

Let $M$ be a hypercomplex manifold, $I$ an induced comlex structure, and $I, J, K \in \mathbb{H}$ the standard triple of induced complex structures. Clearly, $J$ acts 
on the complexified co tangent space $\Lambda^{1} M \otimes \mathbb{C}$ mapping $\Lambda_{I}^{0,1}(M)$ to $\Lambda_{I}^{1,0}(M)$. Consider a differential operator

$$
\partial_{J}: C^{\infty}(M) \longrightarrow \Lambda_{I}^{1,0}(M),
$$

mapping $f$ to $J(\bar{\partial} f)$, where $\bar{\partial}: C^{\infty}(M) \longrightarrow \Lambda_{I}^{0,1}(M)$ is the standard Dolbeault differential on a Kähler manifold $(M, I)$. We extend $\partial_{J}$ to a differential

$$
\partial_{J}: \Lambda_{I}^{p, 0}(M) \longrightarrow \Lambda_{I}^{p+1,0}(M),
$$

using the Leibniz rule.

Proposition 2.2. Let $M$ be a hypercomplex manifold, $I$ an induced complex structure, $I, J, K$ the standard basis in quaternion algebra, and

$$
\Lambda_{+}^{*}(M)=\oplus_{p, q} \Lambda_{I,+}^{p, q}(M)
$$

the Hodge decomposition of the quaternionic Dolbeault complex. Then there exists a canonical isomorphism

$$
\Lambda_{I,+}^{p, q}(M) \cong \Lambda_{I}^{p+q, 0}(M) .
$$

Under this identification, the quaternionic Dolbeault differential

$$
d_{+}: \Lambda_{I,+}^{p, q}(M) \longrightarrow \Lambda_{I,+}^{p+1, q}(M) \oplus \Lambda_{I,+}^{p, q+1}(M)
$$

corresponds to a sum

$$
\partial \oplus \partial_{J}: \Lambda_{I}^{p+q, 0}(M) \longrightarrow \Lambda_{I}^{p+q+1,0}(M) \oplus \Lambda_{I}^{p+q+1,0}(M) .
$$

Proof. This is Proposition 8.13 of [V5].

The statement of Proposition 2.2 can be represented by the following diagram

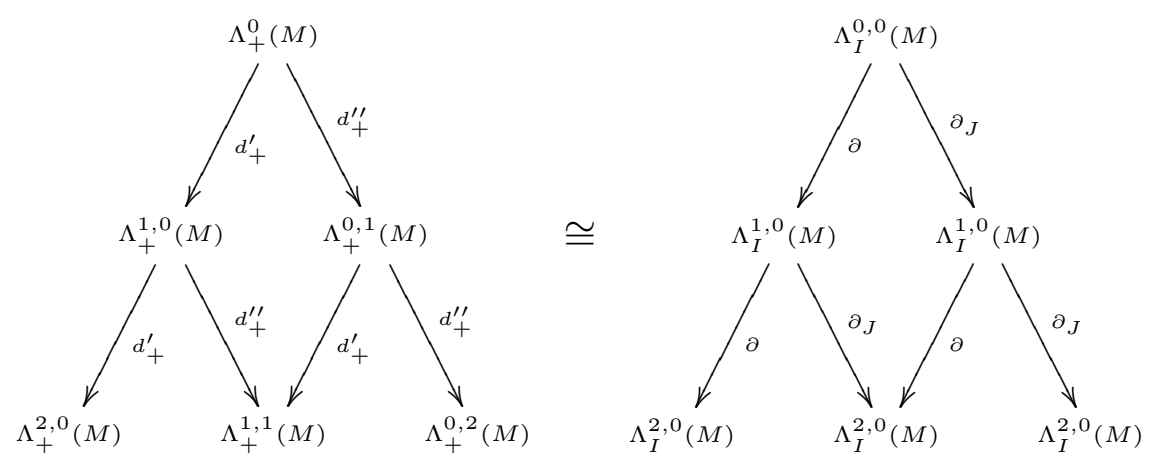

where $d_{+}=d_{+}^{\prime}+d_{+}^{\prime \prime}$ is the Hodge decomposition of the quaternionic Dolbeault differential.

Using the $S U(2)$-action, we may identify the bundles $\Lambda_{+}^{p, q}(M)$ with $\Lambda_{+}^{p+q, 0}(M)$ $=\Lambda_{I}^{p+q, 0}(M)$ explicitly, as follows. 
Let $\mathcal{J}, \mathcal{K}$ be the Lie algebra operators acting on differential forms and associated with $J, K$ in the same way as $\mathcal{I}$ is associated with $I$. Consider the map $\mathcal{R}: \Lambda^{*}(M) \longrightarrow \Lambda^{*}(M)$,

$$
\mathcal{R}:=\frac{\mathcal{J}-\sqrt{-1} \mathcal{K}}{2}
$$

It is easy to check that the Lie algebra elements $\mathcal{R}, \mathcal{I}, \overline{\mathcal{R}}$ form an $S L(2)$-triple in the complexification of the standard $S U(2) \subset \operatorname{End}\left(\Lambda^{*}(M)\right)$. Therefore, $\mathcal{R}$ maps $\Lambda_{+}^{p, q}(M)$ to $\Lambda_{+}^{p+1, q-1}(M)$. Since $\Lambda_{+}^{m}(M)$ is a representation of weight $m$, $\mathcal{R}$ induces an isomorphism

$$
\mathcal{R}: \Lambda_{+, I}^{p, q}(M) \longrightarrow \Lambda_{+, I}^{p+1, q-1}(M),
$$

for all $q>0$.

Together with (2.4), this observation implies the following.

Claim 2.3. Let $M$ be a hypercomplex manifold, $I$ an induced complex structure, and $\eta \in \Lambda_{I}^{1,1}(M)$ a $(1,1)$-form. Then $\eta$ is $S U(2)$-invariant if and only if $\mathcal{R}(\eta)=0$. Moreover, for all functions $\psi$ on $M$, we have

$$
\mathcal{R}(\partial \bar{\partial}(\psi))=\partial \partial_{J}(\psi) .
$$

Assume now that the manifold $M$ is hypercomplex Hermitian. Consider the 3-dimensional space generated by the 2-forms $\omega_{I}, \omega_{J}$ and $\omega_{K}$. This is a weight 2 representationn of $S U(2)$. Moreover, that

$$
\mathcal{R}\left(\omega_{I}\right)=\Omega,
$$

where $\Omega=\frac{1}{2}\left(\omega_{J}+\sqrt{-1} \omega_{K}\right)$ is the canonical $(2,0)$-form.

\section{The $q$-positive forms}

Let $M$ be a hypercomplex manifold, and $\Lambda_{I}^{p, q}(M)$ the bundle of $(p, q)$-forms on $(M, I)$. Consider the map $J: \Lambda^{*}(M) \longrightarrow \Lambda^{*}(M)$,

$$
J\left(d x_{1} \wedge d x_{2} \wedge \ldots\right)=J\left(d x_{1}\right) \wedge J\left(d x_{2}\right) \wedge \ldots
$$

Clearly, on 2-forms we have $J^{2}=1$; more generally,

$$
\left(\left.J\right|_{\Lambda^{\operatorname{even}(M)}}\right)^{2}=1 .
$$

Since $J$ and $I$ anticommute, we have $J\left(\Lambda_{I}^{p, q}(M)\right)=\Lambda_{I}^{q, p}(M)$. By (3.1), the map $\eta \longrightarrow J(\bar{\eta})$ defines a real structure on $\Lambda_{I}^{2,0}(M)$.

Definition 3.1. Let $\eta \in \Lambda_{I}^{2,0}(M)$ be a $(2,0)$-form on a hypercomplex manifold $M$. We say that $\eta$ is $q$-real if $\eta=J(\bar{\eta})$. We say that $\eta$ is $q$-positive if $\eta$ is $q$-real, and

$$
\eta(v, J(\bar{v})) \geqslant 0
$$

for any $v \in T_{I}^{1,0}(M)$. We say that $\eta$ is strictly q-positive if the inequality (3.2) is strict, for all $v \neq 0$. 
The $q$-positive forms were introduced and studied at some length in [V4], under the name " $K$-positive forms". These forms were used to study the stability of certain coherent sheaves. Some properties of $q$-positive forms are remarkably close to that of the usual positive forms, studied in algebraic geometry in connection with Vanishing Theorems.

Proposition 3.2. Let $M$ be a hypercomplex manifold, and $h$ a hypercomplex Hermitian metric. Consider the form

$$
\Omega:=\omega_{J}+\sqrt{-1} \omega_{K}
$$

(see (1.1)). Then $\Omega$ is strictly q-positive. Conversely, every strictly q-positive $(2,0)$-form is obtained from a unique hypercomplex Hermitian metric on $M$.

Proof. The form $\Omega:=\omega_{J}+\sqrt{-1} \omega_{K}$ is $q$-positive as an elementary calculation insures. Indeed, write the orthonormal basis $\xi_{1}, \xi_{2}, \ldots \xi_{2 n} \in \Lambda^{1,0}(M)$ in such a way that

$$
J\left(\xi_{2 i-1}\right)=\bar{\xi}_{2 i}, \quad J\left(\xi_{2 i}\right)=-\bar{\xi}_{2 i-1} .
$$

Then

$$
\Omega=\xi_{1} \wedge \xi_{2}+\xi_{3} \wedge \xi_{4}+\ldots
$$

This form is clearly $q$-real and strictly $q$-positive.

Conversely, let $\Omega$ be a $q$-real and strictly $q$-positive form on a hypercomplex manifold $M$. We can write $\Omega$ is coordinates as

$$
\Omega=\alpha_{1} \xi_{1} \wedge \xi_{2}+\alpha_{3} \xi_{3} \wedge \xi_{4}+\ldots
$$

where $\alpha_{i}$ are positive real numbers, and $\xi_{i}$ satisfy (3.3).

Write a hypercomplex Hermitian form $h$ as

$$
\begin{aligned}
h= & \alpha_{1}\left(\left(\operatorname{Re} \xi_{1}\right)^{2}+\left(\operatorname{Im} \xi_{1}\right)^{2}+\left(\operatorname{Re} \xi_{2}\right)^{2}+\left(\operatorname{Im} \xi_{2}\right)^{2}\right) \\
& +\alpha_{3}\left(\left(\operatorname{Re} \xi_{3}\right)^{2}+\left(\operatorname{Im} \xi_{3}\right)^{2}+\left(\operatorname{Re} \xi_{4}\right)^{2}+\left(\operatorname{Im} \xi_{4}\right)^{2}\right)+\ldots
\end{aligned}
$$

Clearly, the corresponding canonical $(2,0)$-form is equal $\Omega$.

The Hermitian metric (3.5) can be reconstructed from $\Omega$ directly as follows:

$$
h(x, y)=\Omega\left(x^{1,0}, J\left(y^{0,1}\right)\right),
$$

for all $x, y \in T_{\mathbb{R}} M$, where $x^{1,0}, y^{0,1}$ denotes the $(1,0)$ and $(0,1)$-parts of $x, y$. We proved that the hypercomplex Hermitian structure is uniquely determined by the strictly $q$-positive form $\Omega$.

The following Corollary gives an interpretation of HKT-structures in terms of the canonical $(2,0)$-form.

Corollary 3.3. Let $M$ be a hypercomplex manifold, and $\Omega \in \Lambda^{2,0}(M)$ a $\partial$-closed strictly q-positive $(2,0)$-form. Then $M$ is an HKT-manifold, and $\Omega$ is obtained as a canonical $(2,0)$-form of an HKT-metric $h$. Moreover, $h$ is uniquely determined by $\Omega$.

Proof. By Proposition 3.2, $\Omega=\omega_{J}+\sqrt{-1} \Omega_{K}$, for some hypercomplex Hermitian metric $h$. Since $\partial \Omega=0,(M, h)$ is an HKT-manifold. 


\section{Hyperholomorphic bundles}

Hyperholomorphic bundles were introduced and studied at some length in [V1]. Let $B$ be a holomorphic vector bundle over a complex manifold $X, \nabla$ a connection in $B$ and $\Theta \in \Lambda^{2} \otimes \operatorname{End}(B)$ be its curvature. This connection is called compatible with the holomorphic structure if $\nabla_{\gamma}(\zeta)=0$ for any holomorphic section $\zeta$ and any antiholomorphic tangent vector field $\gamma \in T^{0,1}(X)$. If there exists a holomorphic structure compatible with the given Hermitian connection then this connection is called integrable.

Theorem 4.1. Let $\nabla$ be a Hermitian connection in a complex vector bundle $B$ over a complex manifold $X$. Then $\nabla$ is integrable if and only if $\Theta \in \Lambda^{1,1}$ $(X, \operatorname{End}(B))$, where $\Lambda^{1,1}(X, \operatorname{End}(B))$ denotes the forms of Hodge type $(1,1)$. Also, the holomorphic structure compatible with $\nabla$ is unique.

Proof. This is Proposition 4.17 of [Kob], Chapter I.

This proposition is a version of Newlander-Nirenberg theorem. For vector bundles, it was proven by M. Atiyah and R. Bott.

Definition 4.2. Let $B$ be a Hermitian vector bundle with a connection $\nabla$ over a hypercomplex manifold $M$. Then $\nabla$ is called hyperholomorphic if $\nabla$ is integrable with respect to each of the complex structures induced by the hypercomplex structure.

As follows from Theorem 4.1, $\nabla$ is hyperholomorphic if and only if its curvature $\Theta$ is of Hodge type $(1,1)$ with respect to any of the complex structures induced by a hypercomplex structure.

An easy calculation shows that $\nabla$ is hyperholomorphic if and only if $\Theta$ is an $S U(2)$-invariant differential form.

Hyperholomorphic bundles are quite ubiquitous. Clearly, the tangent bundle to a hyperkähler manifold and all its tensor powers are hyperholomorphic. There are many other examples

Example 4.3. Let $M$ be a compact hyperkähler manifold, $B$ a holomorphic bundle. Then $B$ admits a unique hyperholomorphic connection, if $B$ is stable and the cohomology classes $c_{1}(B)$ and $c_{2}(B)$ are $S U(2)$-invariant. Moreover, if $M$ is generic in its deformation class, then all stable bundles admit a hyperholomorphic connection.

\section{5. $\mathbb{H}$-hyperholomorphic bundles}

Definition 5.1. Let $M$ be a hypercomplex manifold, and $(B, \nabla)$ a hyperholomorphic bundle on $M, \operatorname{dim}_{\mathbb{C}} B=2 n$. The bundle $B$ is called $\mathbb{H}$-hyperholomorphic if $\nabla$ preserves a $\mathbb{C}$-linear symplectic structure on $B$. In other words, $B$ is $\mathbb{H}$ hyperholomorphic if the holonomy of $\nabla$ is contained in $S p(n)$.

The following examples are obvious. 
Example 5.2. Let $F$ be a hyperholomorphic bundle on $M$. Then $F \oplus F^{*}$ is $\mathbb{H}$-hyperholomorphic.

Example 5.3. Consider the tangent bundle $T M$ on $M$. Assume that $M$ is hyperkähler. Then $T M$ is $\mathbb{H}$-hyperholomorphic.

The main property of $\mathbb{H}$-hyperholomorphic bundles is the following.

Claim 5.4. Let $M$ be a hypercomplex manifold, and $B$ an $\mathbb{H}$-hyperholomorphic bundle. Denote by Tot $B$ the total space of $B$. Then Tot $B$ is equipped with a natural hypercomplex structure. In particular, the total space of $T M$ is hypercomplex.

Proof. Since the holonomy of $B$ is contained in $S p(n)$, there is a natural parallel action of $\mathbb{H}$ on $B$. Given a quaternion $L \in \mathbb{H}, L^{2}=-1$, consider $B$ as a complex vector bundle with the complex structure defined by $L$. Denote this complex vector bundle as $(B, L)$. Since the curvature of $B$ is $S U(2)$-invariant, the bundle $(B, L)$ is hyperholomorphic. Consider $(B, L)$ as a holomorphic vector bundle on $(M, L)$. Denote the corresponding complex structure on $\operatorname{Tot} B$ by $L$. We obtained an integrable complex structure on Tot $B$ for each quaternion $L \in \mathbb{H}$, $L^{2}=-1$. It is easy to check that these complex structures satisfy quaternionic relations, inducing a hypercomplex structure on Tot $B$.

\section{The Obata connection on Tot $B$.}

Let $M$ be a hyperkähler manifold, and $B$ an $\mathbb{H}$-hyperholomorphic bundle. By Claim 5.4, the total space Tot $B$ is hypercomplex. One can ask whether this hypercomplex structure is hyperkähler. The answer is - never (unless $B$ is flat).

Given a hypercomplex manifold, one can easily establish whether $M$ admits a hyperkähler structure. This is done most easily using the so-called Obata connection.

Theorem 6.1 (Obata). Let $M$ be a hypercomplex manifold. Then $M$ admits a unique torsion-free connection which preserves the hypercomplex structure. ${ }^{1}$

Proof. Well known (see $[\mathrm{Ob}])$.

If $M$ is hyperkähler, then the Levi-Civita connection preserves the hypercomplex structure. In this case, the Levi-Civita connection coincides with the Obata connection.

To determine whether a hypercomplex manifold $M$ admits a hyperkähler structure, one needs to compute the holonomy of the Obata connection. The manifold is hyperkähler if and only if the holonomy Hol preserves a metric; that is, $M$ is hyperkähler if and only if Hol is contained in $S p(n)$.

Proposition 6.2. Let $M$ be a hyperkähler manifold, $B$ an $\mathbb{H}$-hyperholomorphic bundle, and Tot $B$ its total space considered as a hypercomplex manifold (see

\footnotetext{
${ }^{1}$ This connection is called the Obata connection.
} 
Claim 5.4). Assume that the curvature of $B$ is non-zero. Then Tot $B$ does not admit a hyperkähler structure.

Proof. One could compute the holonomy group of the Obata connection of Tot $B$, and show that it is non-compact. To avoid excessive computations, we use a less straightforward argument.

Suppose that Tot $B$ is hyperkähler. Given $m \in M$, let $B_{m} \subset$ Tot $B$ be the fiber of $B$ in $m$. By construction, $B_{m}$ is a hypercomplex submanifold in Tot $B$. Such submanifolds are called trianalytic (see [V2], [V3] for a study of trianalytic cycles on hyperkähler manifolds). In [V3], it was shown that trianalytic submanifolds are completely geodesic. In other words, for any trianalytic submanifold $Z \subset X$, the Levi-Civita connection on $\left.T X\right|_{Z}$ preserves the orthogonal decomposition

$$
\left.T X\right|_{Z}=T Z \oplus T Z^{\perp}
$$

If we have a hypercomplex fibration $X \longrightarrow Y$, the decomposition (6.1) gives a connection for this fibration. In [V3] it was shown that this connection is flat, for any hyperkähler fibration.

We obtain a flat connection $\nabla$ in the fibration $\operatorname{Tot} B \longrightarrow M$. This connection is clearly compatible with the additive structure on the bundle $B$. Therefore, $\nabla$ is an affine connection on $B$. By construction, $\nabla$ is compatible with the hypercomplex structure on Tot $B$. Therefore, $\nabla$ coincides with the hyperholomorphic connection on $B$. We proved that $B$ is flat.

\section{HKT-structure on Tot $B$.}

Let $M$ be a smooth manifold. Given a bundle with connection on $M$, we have a decomposition

$$
T \text { Tot } B=T_{\text {ver }} \oplus T_{\text {hor }}
$$

of the tangent space to Tot $B$ into horizontal and vertical components. Clearly, the bundle $T_{\text {ver }}$ is identified with $\pi^{*} B$, and $T_{\text {hor }}$ with $\pi^{*} T M$, where $\pi$ : Tot $B \longrightarrow$ $M$ is the standard projection.

Assume now that $M$ is a Riemannian manifold, and $B$ a vector bundle, equipped with a Euclidean metric. Then Tot $B$ is equipped with a Riemannian metric $g$ defined by the following conditions.

(i) The decomposition $T$ Tot $B=T_{\text {ver }} \oplus T_{\text {hor }}$ is orthogonal with respect to $g$.

(ii) Under the natural identification $T_{\text {ver }} \cong \pi^{*} B$, the metric $g$ restricted to $T_{\text {ver }}$ becomes the metric on $B$.

(iii) The metric $g$ restricted $T_{\text {hor }} \cong \pi^{*} T M$ is equal to the metric induced on $\pi^{*} T M$ from the Riemannian structure on $M$.

Definition 7.1. In the above assumptions, the metric $g$ is called the natural metric on Tot $B$ induced by the connection and the metrics on $M$ and $B$.

Notice that the metric $g$ depends from the metrics on $B$ and $M$ and from the connection in $B$. Different connections induce different metrics on Tot $B$. 
Theorem 7.2. Let $M$ be an HKT-manifold, and $B$ an $\mathbb{H}$-hyperholomorphic vector bundle on $M$. Consider the metric $g$ on Tot $B$ defined as in Definition 7.1. Then $g$ is an HKT-metric.

Proof. Consider the decomposition $g=\pi^{*} g_{M}+\pi^{*} g_{B}$ of the metric $g$ onto the horizontal and vertical components. Since the decomposition $T$ Tot $B=T_{\text {ver }} \oplus$ $T_{\text {hor }}$ is compatible with the hypercomplex structure, the 2 -forms $g_{\mathrm{hor}}:=\pi^{*} g_{M}$ and $g_{\mathrm{ver}}:=\pi^{*} g_{B}$ are $S U(2)$-invariant. Consider the corresponding (2,0)-forms $\Omega_{\text {hor }}$ and $\Omega_{\text {ver }}$ obtained as in (1.1);

$$
\Omega_{\mathrm{hor}}=\omega_{J \text { hor }}+\sqrt{-1} \omega_{K \text { hor }}
$$

where $\omega_{J \text { hor }}=g_{\text {hor }}(J \cdot, \cdot), \omega_{K \text { hor }}=g_{\text {hor }}(K \cdot, \cdot)$ are differential forms associated with $g_{\text {hor }}$ and $J, K$ as in (1.1).

Then $\Omega_{\text {hor }}$ and $\Omega_{\text {ver }}$ are horizontal and vertical components of the standard $(2,0)$-form of Tot $B$ :

$$
\Omega=\Omega_{\mathrm{hor}}+\Omega_{\mathrm{ver}}
$$

The HKT condition can be written as $\partial \Omega=0$ (1.1). Let $\Omega_{M}$ be the standard $(2,0)$-form of $M$. Since $M$ is an HKT manifold, (1.1) holds on $M$ and the form $\Omega_{\text {hor }}$ satisfies

$$
\partial \Omega_{\mathrm{hor}}=\partial \pi^{*} \Omega_{M}=0 .
$$

To prove Theorem 7.2 , it remains to show

$$
\partial \Omega_{\mathrm{ver}}=0
$$

Consider a function

$$
\Psi: \operatorname{Tot} B \longrightarrow \mathbb{R}, \quad \Psi(v)=|v|^{2},
$$

mapping a vector $v \in T M$ to the square of its norm. Let

$$
0 \longrightarrow \Omega^{1,0} \stackrel{\partial, \partial_{J}}{\longrightarrow} \Omega^{2,0} \stackrel{\partial, \partial_{J}}{\longrightarrow} \Omega^{3,0} \stackrel{\partial, \partial_{J}}{\longrightarrow} \ldots
$$

be the bicomplex defined in (2.4). To prove (7.3), and hence Theorem 7.2 , it suffices to prove

$$
\partial \partial_{J} \Psi=\Omega_{\mathrm{ver}} .
$$

By Claim 2.3, we have

$$
\partial \partial_{J} \Psi=\mathcal{R}(\partial \bar{\partial} \Psi)
$$

where $R: \Lambda^{1,1}(\operatorname{Tot} B) \longrightarrow \Lambda^{2,0}(\operatorname{Tot} B)$ is the operator

$$
\mathcal{R}=\frac{\mathcal{J}-\sqrt{-1} \mathcal{K}}{2}
$$

(see $(2.5))$. However, the 2 -form $\partial \bar{\partial} \Psi$ is quite easy to compute. From [Bes], (15.19), we obtain:

$$
\partial \bar{\partial} \Psi=\omega_{\mathrm{ver}}+\xi
$$


where $\omega_{\text {ver }}=g_{\text {ver }}(\cdot, I \cdot)$ is the Hermitian form of $g_{\text {ver }}$, and $\xi$ is defined as following. Using the decomposition (7.1), we consider $\Lambda^{2} T_{\text {hor }}$ as a subbundle in $\Lambda^{2} \operatorname{Tot} B$. Then $\xi \in \Lambda^{2} T_{\text {hor }} \subset \Lambda^{2}$ Tot $B$ is a 2 -form on $T_{\text {hor }}$ mapping a pair of vectors $(x, y)$

$$
\begin{aligned}
x, y \in & \left.T_{\text {hor }}\right|_{(m, b)} \subset T_{(m, b)} \operatorname{Tot} B, \\
& \left.T_{\text {hor }}\right|_{(m, b)}=T_{m} M, \\
(m, b) \in & \operatorname{Tot} B, m \in M,\left.b \in B\right|_{m}
\end{aligned}
$$

to $(R(x, y, b) \bar{b})$, where $R \in \Lambda^{2} M \otimes$ End $B$ is the curvature of $B$. The form $\xi$ is $S U(2)$-invariant because the curvature of $B$ is $S U(2)$-invariant. Therefore, $\mathcal{R}(\xi)=0$ (Claim 2.3), and

$$
\partial \partial_{J} \Psi=\mathcal{R}(\partial \bar{\partial} \Psi)=\mathcal{R}\left(\omega_{\text {ver }}\right)=\Omega_{\text {ver }}
$$

(the last equation holds by (2.6)). This proves (7.5). Theorem 7.2 is proven.

\section{New examples of compact HKT-manifolds}

Let $M$ be a compact HKT-manifold, e.g. a hyperkähler manifold, and $B$ an $\mathbb{H}$-hyperholomorphic vector bundle on $M$ (for examples of $\mathbb{H}$-hyperholomorphic vector bundles see Examples 5.2 and 5.3). Denote by $\operatorname{Tot}^{\circ} B$ be the space of non-zero vectors in $B$. Fix a real number $q>1$. Consider the map

$$
\rho_{q}: \operatorname{Tot}^{\circ} B \longrightarrow \operatorname{Tot}^{\circ} B, \quad \rho_{q}(b)=q b, \quad b \in \operatorname{Tot}^{\circ} B,
$$

and let $\mathcal{M}=\operatorname{Tot}^{\circ} B / \rho_{q}$ be the corresponding quotient space. Since the map $b \longrightarrow q b$ is compatible with the hypercomplex structure, the space $\mathcal{M}$ is hypercomplex. It is fibered over a compact manifold $M$, with fibers Hopf manifolds which are homeomorphic to $S^{1} \times S^{2 m-1}, m=\operatorname{dim}_{\mathbb{R}} B$, hence it is compact.

Theorem 8.1. In the above assumptions, $\mathcal{M}$ admits a natural HKT-structure.

Proof. By Corollary 3.3, we need to construct a $q$-positive $\partial$-closed $(2,0)$-form on $\mathcal{M}$. Let $\widetilde{\Omega}$ be a $(2,0)$-form on $\operatorname{Tot}^{\circ} B$,

$$
\widetilde{\Omega}=\pi^{*} \Omega_{M}+\partial \partial_{J} \log \Psi,
$$

where $\pi^{*} \Omega_{M}$ is the canonical $(2,0)$-form on $M$ lifted to $\operatorname{Tot}^{\circ} B$, and $\Psi$ : $\operatorname{Tot} B \longrightarrow$ $\mathbb{R}$ the square norm function (7.4). The map $v \stackrel{\rho_{q}}{\longrightarrow} q v$ satisfies $\rho_{q}^{*} \log \Psi=$ $\log \Psi+\log q^{2}$, and therefore

$$
\rho_{q}^{*} \partial \partial_{J} \log \Psi=\partial \partial_{J} \log \Psi
$$

This implies that $\widetilde{\Omega}=\pi^{*} \Omega_{M}+\partial \partial_{J} \log \Psi$ is $\rho_{q}$-invariant, hence defines a form $\Omega$ on $\mathcal{M}=\operatorname{Tot}^{\circ} B / \rho_{q}$.

By construction, the form $\Omega$ is $\partial$-closed. To prove Theorem 8.1, it remains to show that $\widetilde{\Omega}$ is strictly $q$-positive. We use the same argument as used to show that a locally conformal hyperkähler manifold is HKT. 
We have

$$
\partial \partial_{J} \log \Psi=\frac{\partial \partial_{J} \Psi}{\Psi}-\frac{\partial \Psi \wedge \partial_{J} \Psi}{\Psi^{2}} .
$$

In all directions orthogonal to $\partial \Psi, \partial_{J} \Psi$, the form $\partial \partial_{J} \log \Psi$ is proportional to $\partial \partial_{J} \Psi$, hence $q$-positive by (7.7). Moreover, (8.1) implies that

$$
\widetilde{\Omega}=\Omega_{\mathrm{hor}}+\frac{\Omega_{\mathrm{ver}}}{\Psi}-\frac{\partial \Psi \wedge \partial_{J} \Psi}{\Psi^{2}},
$$

(we use the notation introduced in Section 7 ). The form $\Omega_{\text {hor }}+\frac{\Omega_{\text {ver }}}{\Psi}$ is strictly $q$-positive (Theorem 7.2). The vertical and the horizontal tangent vectors are orthogonal with respect to $\widetilde{\Omega}$. Since $\frac{\partial \Psi \wedge \partial_{J} \Psi}{\Psi^{2}}$ vanishes on all horizontal tangent vectors, it remains to prove that $\widetilde{\Omega}(x, J \bar{x})>0$, where $x$ is vertical.

Let $\xi \in T^{1,0}\left(\operatorname{Tot}^{\circ} B\right)$ be the vertical tangent vector to $\operatorname{Tot}^{\circ} B$ which is dual to $\frac{d \Psi}{\sqrt{\Psi}}$. Clearly, $\partial \Psi$ is the $(1,0)$-part of $\xi$. For all $x \in T^{1,0}\left(\operatorname{Tot}^{\circ} B\right)$, we have

$$
\frac{\partial \Psi \wedge \partial_{J} \Psi}{\Psi^{2}}(x, J(\bar{x}))=(\xi, x)_{H}^{2},
$$

where $(\cdot, \cdot)_{H}$ denotes the Riemannian form. Similarly,

$$
\Omega_{\mathrm{ver}}(x, J(\bar{x}))=2(x, x)_{H}
$$

(this can be checked by writing $\Omega_{\mathrm{ver}}$ is coordinates as in (3.4)). Using Cauchy inequality and $|\xi|=1$, we obtain $(x, x)_{H} \geqslant(\xi, x)_{H}^{2}$. Then

$$
\begin{aligned}
\widetilde{\Omega}(x, J(\bar{x})) & =\frac{\Omega_{\mathrm{ver}}}{\Psi}(x, J(\bar{x}))-\frac{\partial \Psi \wedge \partial_{J} \Psi}{\Psi^{2}}(x, J(\bar{x})) \\
& =2 \frac{(x, x)_{H}}{\Psi}-\frac{(\xi, x)_{H}^{2}}{\Psi} \geqslant \frac{(x, x)_{H}}{\Psi}>0
\end{aligned}
$$

for all vertical tangent vectors $x \neq 0$. This proves Theorem 8.1.

\section{Acknowledgements}

I am grateful to G. Grantcharov, D. Kaledin and Y.S. Poon for interesting discussions.

\section{References}

[Bes] A. Besse, Einstein manifolds, Ergebnisse der Mathematik und ihrer Grenzgebiete (3), 10. Springer-Verlag, Berlin, 1987.

[Bo] C. P. Boyer, A note on hyper-Hermitian four-manifolds, Proc. Amer. Math. Soc. 102 (1988), 157-164.

[Ca] E. Calabi, Métriques kählériennes et fibrés holomorphes, Ann. Sci. École Norm. Sup. (4) 12 (1979), 269-294.

[FG] A. Fino, G. Grantcharov, On some properties of the manifolds with skew-symmetric torsion and holonomy $S U(n)$ and $S p(n)$, preprint: math.DG/0302358

[GP] G. Grantcharov, Y.S. Poon, Geometry of hyper-Kähler connections with torsion, Comm. Math. Phys. 213 (2000), 19-37.

[GH] P. Griffiths, J. Harris, Principles of algebraic geometry, Wiley-Intersience, New York, 1978. 
[GP1] J. Gutowski, G. Papadopoulos, The dynamics of very special black holes, Phys. Lett. B 472 (2000), 45-53.

[GP2] _ The moduli spaces of worldvolume brane solitons, Phys. Lett. B 432 (1998), 97-102.

[GPS] G. W. Gibbons, G. Papadopoulos, K.S. Stelle, HKT and OKT geometries on soliton black hole moduli spaces, Nuclear Phys. B 508 (1997), 623-658.

[HP] P.S. Howe, G. Papadopoulos, Twistor spaces for hyper-Kähler manifolds with torsion, Phys. Lett. B 379 (1996), 80-86.

[I] S. Ivanov, Geometry of quaternionic Kähler connections with torsion, J. Geom. Phys. 41 (2002), 235-257; math.DG/0003214

[J] D. Joyce, Compact hypercomplex and quaternionic manifolds, J. Differential Geom. 35 (1992), 743-761.

[Kob] S. Kobayashi, Differential geometry of complex vector bundles, Princeton University Press, Princeton, NJ, 1987.

[Ob] M. Obata, Affine connections on manifolds with almost complex, quaternion or Hermitian structure, Jap. J. Math. 26 (1956), 43-77.

[Or] L. Ornea, Weyl structures on quaternionic manifolds. A state of the art, preprint: math.DG/0105041

[OPS] L. Ornea, Y.S. Poon, A. Swann, Potential one-forms for hyperkähler structures with torsion, preprint: math.DG/0211427

[P] G. Papadopoulos, Brane Solitons and Hypercomplex Structures, Quaternionic structures in mathematics and physics (Rome, 1999), 299-312; math.DG/0003024

[PP] H. Pedersen, Y.S. Poon, Inhomogeneous hypercomplex structures on homogeneous manifolds, J. Reine Angew. Math. 516 (1999), 159-181.

[V1] M. Verbitsky, Hyperholomorphic bundles over a hyper-Kähler manifold, J. Algebraic Geom. 5 (1996), 633-669; alg-geom/9307008

[V2] - Tri-analytic subvarieties of hyper-Kaehler manifolds, Geom. Funct. Anal. 5 (1995), 92-104; Also available as Hyperkähler embeddings and holomorphic symplectic geometry. $I I$ : alg-geom/9403006

[V3] _ Deformations of trianalytic subvarieties of hyper-Kähler manifolds, Selecta Math. (N.S.) 4 (1998), 447-490; alg-geom/9610010

[V4] - Hyperholomorpic connections on coherent sheaves and stability, preprint: math. AG/0107182

[V5] _ Projective bundles over hyperkähler manifolds and stability of Fourier-Mukai transform, preprint: math.AG/0107196

[V6] - HyperKähler manifolds with torsion, supersymmetry and Hodge theory, Asian J. Math. 6 (2002), 679-712; math.AG/0112215

Independent University of Moscow, Bolshoi Vlasevsky Pereulok, 11, Moscow, 119002, Russia.

E-mail address: verbit@mccme.ru

Glasgow University, Deptartment of Mathematics, 15 University gardens, Glasgow, G12 8QW, Scotland.

E-mail address: verbit@maths.gla.ac.uk 